Abstract P192 Table 1 Delegates choosing GUM as a speciality following taster day

\begin{tabular}{ll}
\hline Specialty & $\begin{array}{l}\text { Number of trainees } \\
\text { (\% choosing specialty) }\end{array}$ \\
\hline GUM & $17(36)$ \\
GP & $10(21)$ \\
Microbiology/radiology & 3 each (6) \\
ID/Public health/Surgery/O\&G & 2 each (4) \\
Dermatology/oncology/geriatrics/rheumatology/ACCS/psychiatry & 1 each (2) \\
Total & 47 \\
\hline
\end{tabular}

\section{P193 A MIXED-METHOD STUDY OF HOW TO INCREASE STI SCREENING AMONG YOUNG PEOPLE}

doi:10.1136/sextrans-2012-050601c.193

${ }^{1} \mathrm{~N}$ Loades, ${ }^{*}{ }^{2} \mathrm{R}$ de Visser. ${ }^{1}$ Brighton and Sussex Medical School, Brighton, UK; ${ }^{2}$ University of Sussex, Brighton, UK

Background STIs are a considerable problem, with diagnoses concentrated among young people. Although the Theory of Planned Behaviour (TPB) predicts many health behaviours, there is a lack of research into how well it explains STI testing. The original TPB emphasised the importance of intentions as the ultimate influence on behaviour. Subsequent research focuses on implementation intentions-people who make explicit plans for how to implement their intentions are more likely to do so. Although some studies have analysed barriers and facilitators in STI testing, no published research has examined what impact developing implementation plans has. This study was designed to address this knowledge gap.

Objectives To assess an extended TPB and implementation intention interventions on STI testing; to carry out a qualitative study of barriers and facilitators in those who intend to test.

Methods 371 participants completed an online baseline questionnaire and were randomly allocated to a control, information or implementation plan condition to examine differential effects on intentions to undergo STI testing. 172 completed a 6-week followup questionnaire to assess actual testing behaviour. In-depth interviews were conducted with a purposive selection of the sample and analysed using interpretative phenomenological analysis.

Results Multivariate analysis revealed that TPB variables explained $20 \%$ of the variance in past testing behaviour $(p<0.01)$ and $17 \%$ of variance in intention $(p<0.01)$. No significant difference between intervention arms was found $(p=0.90)$ (the intervention did not significantly strengthen intentions). Qualitative analysis revealed multiple perceived barriers to STI testing.

Conclusion Results provide support for the utility of an extended TPB in predicting past behaviour and intentions to undergo STI testing. Although the implementation plan intervention was ineffective, qualitative data helped explain why a significant intervention effect was not found.

\section{P194 THE USE OF CAPTURE RE-CAPTURE TO ASSESS COMPLETENESS OF REPORTING OF SYPHILIS CASES AND TO GUIDE INTERVENTIONS FOR IMPROVEMENT}

doi:10.1136/sextrans-2012-050601c.194

\section{A Waldram, ${ }^{*}$ R Gorton. Health Protection Agency}

Background Two North East Syphilis outbreaks underlined the need for complete reporting of cases. Completeness was assessed by comparing GUMCAD with regional Enhanced Syphilis Surveillance. Capture-recapture studies were undertaken at yearly intervals to estimate the true number of cases and put forward recommendations to improve reporting completeness.

Objectives The objectives were to assess completeness of reporting of Syphilis cases in two surveillance systems, estimate the true number of cases, and provide recommendations for improving Syphilis reporting. Methods Completeness of reporting was assessed for the period January 2010-June 2010 using capture-recapture methodology to estimate the true number of cases. Intervention A (clinic visits discussing findings) was performed and the effect measured by repeating the process. Follow-up studies were undertaken covering January 2010 to Jun 2011 to measure the effect and a different intervention B (linelists sent to clinics) was performed.

Results In the initial audit neither system included more than $73 \%$ of the estimated true burden of cases, although the aggregate figures suggested they were similar. After intervention A this increased to $94 \%$ in GUMCAD. The second audit showed a decreasing trend of reporting completeness after June 2010 to an average of $84 \%$ in January 2010-June 2011. After intervention B this improved to $96 \%$. Causes of incompleteness in GUMCAD included incorrect coding and failure to record cases identified during screening leading to under-matching and overestimation of the total burden.

Discussion Completeness of reporting before intervention was $84 \%$ in GUMCAD suggesting the true number of syphilis cases was 19\% higher than reported by official statistics. Completeness increased after intervention A but subsequently declined and a further intervention was required to maintain data quality. Regular feedback of case lists to clinics to check for missing cases can enhance completeness of Syphilis surveillance.

\section{P195 ASKING ABOUT SEX IN GENERAL HEALTH SURVEYS: IT CAN BE DONE! COMPARING DATA COLLECTED BY THE 2010 HEALTH SURVEY FOR ENGLAND WITH NATSAL}

doi:10.1136/sextrans-2012-050601c.195

${ }^{1} \mathrm{C}$ H Mercer, ${ }^{*}{ }^{2} \mathrm{C}$ Robinson, ${ }^{2} \mathrm{R}$ Craig, ${ }^{3} \mathrm{~A}$ Nardone, ${ }^{1} \mathrm{~A}$ M Johnson. ${ }^{1}$ University College London, London, UK; ${ }^{2}$ National Centre for Social Research; ${ }^{3}$ Health Protection Agency

Objectives The HSE is administered annually to a probability sample of people in England. In 2010, the HSE included, for the first time, questions about sexual health, which previously were considered too sensitive for a general health survey. This paper compares the reporting of sexual behaviours by people aged 16-44 in HSE2010 with Natsal-2, Britain's national probability survey of sexual behaviour.

Methods HSE2010 interviewed 8420 people aged 16-69, of whom 2911 were $16-44$. Natsal-2 interviewed 12110 people aged 16-44 in 1999/2001. HSE2010 used pen-and-paper self-completion questionnaires for the sexual health questions, while Natsal-2 used computer-assisted self-interviews for the more sensitive questions. Results Collecting sexual behaviour data were acceptable to HSE2010 participants with low item non-response $(5 \%-10 \%)$, albeit slightly higher than in Natsal-2 $(<5 \%)$. Reported age at 1 st intercourse was comparable in the two surveys: medians of 17 (men) and 16 (women) aged 16-24. However, for some very sensitive questions there were lower levels of reporting in HSE2010 than in Natsal-2: while the proportion reporting same-sex in the last 5 years was similar $(2 \%-3 \%)$, reporting of ever having same-sex was lower in HSE2010 for men (2\% vs $5 \%$ in Natsal-2). Similarly, the mean number of opposite-sex partners reported in HSE2010 was a little lower than in Natsal-2, particularly for men: 9.5 vs 12.7, respectively; (5.4 vs 6.5 for women, respectively). Men were also slightly less likely to report STI diagnoses in HSE2010 vs Natsal-2: $8.7 \%$ vs $11.1 \%$, respectively; but this was reported by similar proportions of women (12\%).

Discussion Sexual behaviour data can be successfully collected by the HSE, albeit in less detail than in Natsal. HSE2010 has 
demonstrated the feasibility and utility of including such questions in general health surveys, providing a useful vehicle for monitoring sexual risk behaviour more frequently than is possible with decennial Natsal surveys.

\section{P196 SERVICE EVALUATION: SEXUAL HEALTH PROMOTION A "MISSED" OPPORTUNITY FOR YOUNG PEOPLE?}

doi:10.1136/sextrans-2012-050601c.196

${ }^{1} \mathrm{C}$ Lutterodt, ${ }^{2} \mathrm{~L}$ Wong-Taylor, ${ }^{2} \mathrm{~B}$ White, ${ }^{2} \mathrm{G}$ Lewis. ${ }^{1} 75$ London road, London, UK; ${ }^{2}$ University College Hospital, London, UK

Background There is currently no widely accepted guidelines regarding sexual health promotion for paediatric staff. Consequently, valuable opportunities to provide much needed education are unfortunately being "missed". Young people (YP) in hospital have an ideal opportunity to discuss sexual health in confidence with healthcare workers.

Objectives The objective of this service evaluation was to assess the role of healthcare professionals and their views on providing sexual health education to adolescents on the wards. Understanding perceived views and identifying barriers and leavers is crucial in exploring some of the reasons behind why potentially ideal situations to provide sexual health information are currently being "missed".

Methods Questionnaires were completed by paediatric staff members. The responses were anonymised and data were collected from mid-October 2011 until the end January 2012.

Results The overall response rate was $81 \%$. The skills paediatric staff felt they possessed were: advising how to protect against pregnancy (91\%), STDs (90\%), prescribing contraception (61\%), screening for psychosocial issues (26\%), testing (34\%) and treating STDs (40\%). Almost $20 \%$ did not know about local sexual health centres. Furthermore, GPs were cited as the most appropriate healthcare professional to provide sexual health information.

Conclusion There appears to be a role for sexual health education within secondary care. We suggest that opportunistic health promotion should be considered by paediatric staff when dealing with YPs. Clearer guidance would minimise the number of "missed" opportunities and additional information sources (eg, leaflets) for patients and staff would enhance the provision of health promotion.

\section{P197 \\ YOUNG ASIAN SEXUAL HEALTH: WHAT CAN YOUNG INDIAN, PAKISTANI AND BANGLADESHI PEOPLE TELL US ABOUT THEIR BELIEFS, ATTITUDES AND BEHAVIOURS REGARDING SEXUAL HEALTH AND SEX AND RELATIONSHIPS EDUCATION?}

doi:10.1136/sextrans-2012-050601c.197

J Reynolds-Wright, ${ }^{*}$ N Alice, M Michelle, H Kanabar. University of Sheffield, Sheffield, UK

Introduction Young people's sexual health in the UK has been a concern for public health policy and, despite falling teenage pregnancy rates, sexually transmitted infections (STIs) among young people have shown an increase. The numbers of Asian teenagers engaging in sexual intercourse is reportedly lower than their Black counterparts, however, there is some evidence that Asian young people are more likely to report "regretful intercourse", "unequal willingness" and higher rates of anal intercourse, and so are still at risk of STIs through their behaviour.

Methods 16-25-year-old South Asian people were recruited from schools, universities and community organisations to participate in focus group (FG) or individual interviews. The Topic guide was developed following a literature review, steering group discussions and pilot FG. Following each FG or interview, emergent themes were explored in subsequent discussions. Ethical Permission was granted by NHS South Yorkshire Research Ethics Committee.

Results NVivo 8 software was used to code, organise and support thematic analysis. A total of five FGs and six interviews have been completed and analysis indicates five over-arching themes: (1). Formal sex and relationships education (SRE) (2). Informal SRE (3). Relationships (4). Barriers to access of SRE and sexual health services (5). Future development

Conclusion South Asian young people have distinct health education needs that are underpinned and informed by their ethnic, religious and cultural background. In order to deliver the best level of care to this group, awareness of these needs is essential. 